\title{
Muscular Ultrasound, Syndecan-1 and Procalcitonin Serum Levels to Assess Intensive Care Unit-Acquired Weakness
}

\author{
Robert Patejdl ${ }^{+}$, Uwe Walter ${ }^{+}$, Sarah Rosener, Martin Sauer, Daniel A. Reuter, \\ Johannes Ehler (D)
}

\begin{abstract}
Background: Intensive care unit-acquired weakness (ICU-AW) is associated with poorer outcome of critically ill patients. Microcirculatory changes and altered vascular permeability of skeletal muscles might contribute to the pathogenesis of ICU-AW. Muscular ultrasound (MUS) displays increased muscle echogenicity, although its pathogenesis is uncertain. Objective: We investigated the combined measurement of serum and ultrasound markers to assess ICU-AW and clinical patient outcome. Methods: Fifteen patients and five healthy controls were longitudinally assessed for signs of ICU-AW at study days 3 and 10 using a muscle strength sum score. The definition of ICU-AW was based on decreased muscle strength assessed by the muscular research council-sum score. Ultrasound echogenicity of extremity muscles was assessed using a standardized protocol. Serum markers of inflammation and endothelial damage were measured. The 3-month outcome was assessed on the modified Rankin scale. Results: ICU-AW was present in eight patients, and seven patients and the control subjects did not develop ICU-AW. The global muscle echogenicity score (GME) differed significantly between controls and patients (mean GME, $1.1 \pm 0.06$ vs. $2.3 \pm 0.41 ; p=0.001$ ). Mean GME values significantly decreased in patients without ICU-AW from assessment $1(2.30 \pm 0.48)$ to assessment $2(2.06 \pm 0.45 ; p=0.027)$, which was not observed in patients with ICU-AW. Serum levels of syndecan-1 at day 3 significantly correlated with higher GME values at day $10(r=0.63, p=0.012)$. Furthermore, the patients' GME significantly correlated with $\mathrm{mRS}$ at day $100(r=0.67, p=0.013)$. Conclusion: The combined use of muscular ultrasound and inflammatory biomarkers might be helpful to diagnose ICU-AW and to predict long-term outcome in critical illness.
\end{abstract}

RÉSUMÉ: Utiliser l'échographie des muscles et les niveaux sériques de CD138 et de procalcitonine pour évaluer des patients atteints du syndrome de faiblesse acquise aux soins intensifs. Contexte: Le syndrome de faiblesse acquise aux soins intensifs est associé, chez des patients gravement malades, à une évolution davantage défavorable de leur état de santé. À cet effet, il est possible que des modifications de nature microcirculatoire ainsi qu'une perméabilité vasculaire altérée des muscles squelettiques contribuent à la pathogénèse de ce syndrome. Des échographies des muscles peuvent certes indiquer une échogénicité accrue des muscles ; cela dit, la pathogénèse de ce signe clinique demeure incertaine. Objectif: Nous avons analysé la capacité combinée de mesure d'un sérum et de marqueurs utilisés lors d'échographies afin d'évaluer, chez des patients, le syndrome de faiblesse acquise aux soins intensifs ainsi que l'évolution de leur état de santé. Méthodes: Aux jours 3 et 10 de cette étude, quinze patients et cinq témoins en santé ont été évalués de façon longitudinale afin de détecter des signes de faiblesse, et ce, en utilisant un score global mesurant leur force musculaire : le Muscular Research Council-Sum Score (MRC-SS). L'échogénicité de l'extrémité des muscles a été mesurée au moyen d'un protocole normalisé. De plus, des marqueurs sériques d'inflammation et de lésions endothéliales ont été eux aussi mesurés. Au bout de 3 mois, l'évolution de l'état de santé des patients a été évaluée à l'aide de l'échelle de Rankin modifiée. Résultats: Le syndrome de faiblesse acquise aux soins intensifs s'est révélé présent chez huit patients; aucun témoin ne l'a par ailleurs développé. Le score global d'échogénicité des muscles (SGEM) des témoins a différé de façon notable de celui des patients (SGEM moyen 1,1 $\pm 0,06$ contre 2,3 $\pm 0,41 ; p=0,001$ ). Les valeurs moyennes au SGEM ont par ailleurs diminué de façon significative chez les patients non atteints par ce syndrome si on compare le jour $3(2,30 \pm 0,48)$ au jour $10(2,06 \pm 0,45 ; p=0,027)$, ce qui n'a pas été observé chez les patients qui en étaient atteints. Les niveaux sériques de protéine CD138 au jour 3 sont apparus étroitement liés à des valeurs plus élevées au SGEM au jour $10(r=0,63 ; p=0,012)$. En terminant, soulignons que le SGEM des patients est apparu nettement corrélé à l'échelle de Rankin modifiée au jour 100 ( $r=0,67 ; p=0,013)$. Conclusion: L'utilisation combinée d'échographies des muscles et de biomarqueurs inflammatoires pourrait donc s'avérer utile pour diagnostiquer le syndrome de faiblesse acquise aux soins intensifs et prédire l'évolution de santé à long terme de patients atteints de graves maladies.

Keywords: Intensive care unit, Critical illness polyneuropathy, Critical illness myopathy, Neuromuscular dysfunction, Muscular ultrasound, Electrophysiology, Diagnosis

doi: $10.1017 / \operatorname{cjn} .2018 .390$

Can J Neurol Sci. 2019; 46: 234-242

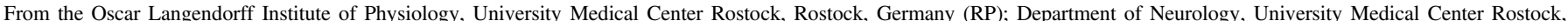

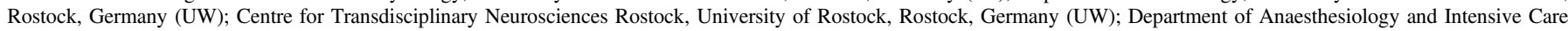
Medicine, University Medical, Center Rostock, Rostock, Germany (SR, MS, DAR, JE)

Received October 11, 2018. Final Revisions Submitted November 27, 2018. Date of Acceptance December 8, 2018.

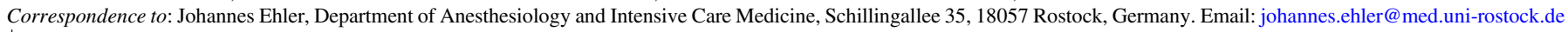
${ }^{+}$These authors contributed equally. 


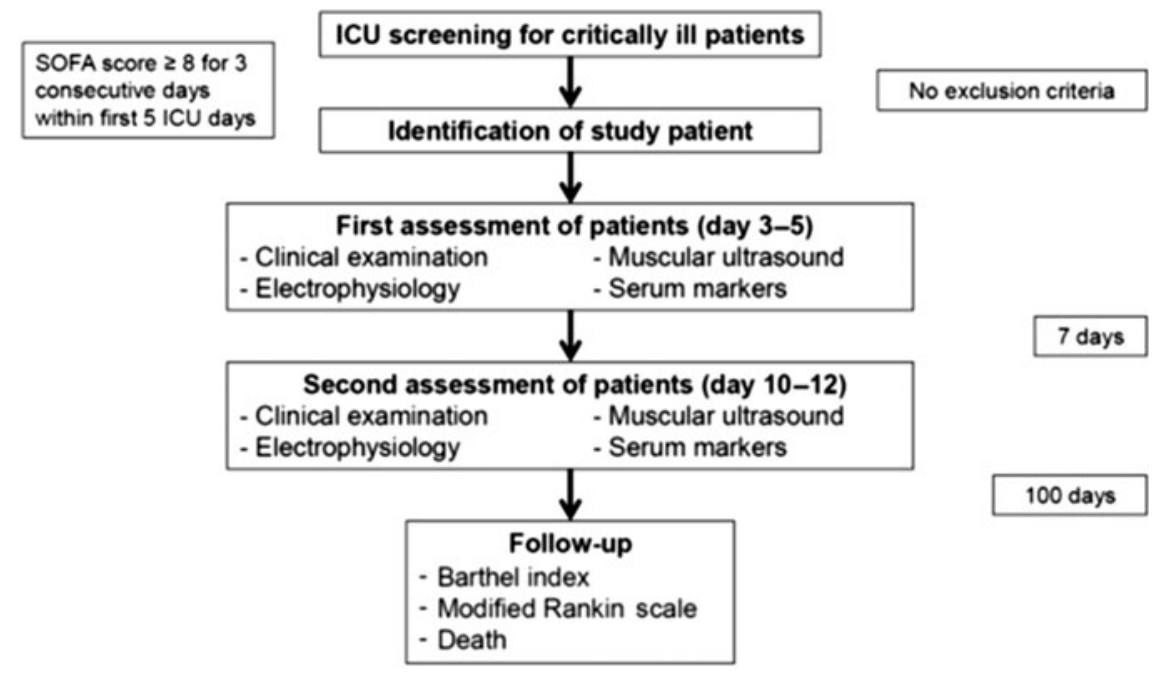

Figure 1: Study protocol for the standardized multimodal assessment of patients and controls. $I C U$, intensive care unit; SOFA, sequential organ failure assessment.

\section{INTRODUCTION}

Intensive care unit-acquired weakness (ICU-AW) is recognized as a frequent complication of critical illness. ${ }^{1,2}$ Approximately $50 \%$ of critically ill patients develop ICU-AW which is associated with prolonged mechanical ventilation, increased ICU stay, higher mortality rates, and reduced quality of life of survivors. ${ }^{1-4}$ Stevens et al. proposed the term ICU-AW for a clinically detected weakness in critically ill patients that has no other reasons for neuromuscular alterations except critical illness. ${ }^{2,4}$ Risk factors like inflammation, disease severity, sedation, use of neuromuscular blocking agents, or the administration of glucocorticosteroids are emerging and point towards a multifactorial pathogenesis of ICU-AW. ${ }^{5-7}$ Recent studies gave evidence for an early induction of systemic inflammatory pathways in ICU-AW. ${ }^{3,6,8,9}$ Other studies focused on an inflammation-mediated degradation of the endothelial glycokalyx, a complex macromolecular network involved in endothelial functions in sepsis and neuromuscular disorders. ${ }^{10-12}$ Increased e-selectin, syndecan-1, and cytokine levels were linked to endothelial damage and neuromuscular complications in critical illness. ${ }^{3,12-14}$ Diagnosis and monitoring of ICU-AW is challenging in sedated and ventilated ICU patients as the clinical examination requires cooperative patients which represents a typical shortcoming in the assessment. ${ }^{15,16}$ In contrast, muscular ultrasound (MUS) is independent from patient cooperation and is a painless, non-invasive, and commonly available bedside technique with emerging relevance for the diagnosis and monitoring of neuromuscular disorders in critically ill patients. ${ }^{15-18}$ Increased echogenicity of skeletal muscles was observed by MUS in ICU patients and was correlated with neuromuscular dysfunction. ${ }^{19-22}$ The pathogenesis of increased muscle echogenicity is uncertain. ${ }^{16}$ Inflammatory myopathic changes resulting in capillary leakage, edema, myofascial inflammation, and myofiber necrosis are discussed. ${ }^{15,16,19,23}$

We conducted a prospective and longitudinal pilot observational study to investigate the relationship between increased muscle echogenicity, serum markers of inflammation and endothelial damage and their potential relevance for ICU-AW and outcome in critically ill patients.

\section{Methods}

\section{Study Setting and Approvals}

This study was performed on two perioperative ICUs of the University Medical Center Rostock, Germany. It was approved by the local ethics committee of Rostock University (identifier: A 2016-0016) and was registered as a clinical trial (ClinicalTrials. gov: NCT02706314). Written informed consent was obtained from all participants or their legal representatives prior to study inclusion. All data were anonymized prior to the analysis.

\section{Inclusion and Exclusion Criteria}

Critically ill ICU patients at ages $\geq 18$ years, who had to be admitted with a sequential organ failure assessment score $\geq 8$ on three consecutive days within the first 5 ICU days were eligible for study inclusion. Exclusion criteria were a history of any neuromuscular disorder and a pre-existing medication with glucocorticosteroids. In addition, healthy and age-matched controls were prospectively included. An overview about patient screening and the study protocol is shown in Figure 1.

\section{Clinical Assessment of Critical Illness and Long-Term Follow-Up for 100 Days}

All patients were clinically assessed by a physician experienced in critical and neurocritical care (JE). The Acute Physiology and Chronic Health Evaluation-II-score at the time of ICU admission and the sequential organ failure assessment score at the time of first and second assessments were ascertained from all patients. $^{24,25}$ The level of consciousness was evaluated using the Glasgow Coma Scale and the Richmond Agitation and Sedation Scale. ${ }^{26,27}$ The Barthel index (BI, activities of daily living) and the modified Rankin scale (mRS) before hospital admission were determined based on information obtained from hospital records and by interview with relatives. All patients and controls were screened for risk factors for polyneuromyopathy like diabetes mellitus, alcohol abuse, vitamin deficiency, renal insufficiency/ replacement therapy, or chemotherapy. ${ }^{28}$ Serum measurements of vitamin B1, B6, B12, folic acid, homocysteine, hemoglobin A1c 
levels, and an immunofixation screening for paraproteins were performed after study inclusion in all patients to exclude any potential impact of previously undetected polyneuromyopathy. In all patients, length of ICU and hospital stay, days on ventilator, days and cumulative dosage of catecholamines, 28- and 100-day mortality were assessed. The BI and the mRS were ascertained by a standardized telephone interview with the patients or their legal representative at day 100 after study inclusion.

\section{Clinical Assessment of Neuromuscular Function and Diagnosis of ICU-AW}

A standardized clinical neurological examination was performed at the time of first and second clinical assessments in all patients by an intensivist experienced in critical and neurocritical care (JE) who was blinded to the results from MUS and electrophysiology. Patients on continuous analgosedation received a sedation holiday for at least 2 hours before each examination. Brainstem function, deep tendon reflexes, sensory and motor function were examined. ICU-AW was defined as a diffuse, symmetric, and flaccid weakness without involvement of cranial nerves. ${ }^{2}$ Muscle strength was assessed using the previously validated Medical Research Council-Sum score (MRC-SS). ${ }^{2,7,29}$ ICU-AW was regarded as being present at an MRC-SS of $<48$ points. $^{2,29}$ The conclusive allocation of patients to the ICU-AW positive and negative group was based on the MRC-SS evaluation at the time of assessment 2. If patients were unable to follow all five standardized commands due to analgosedation or delirium, the MRC-SS was evaluated as "unable-to-assess" according to recommendations. ${ }^{29}$ The administration of neuromuscular blocking agents within 24 hours before clinical examination was excluded.

\section{Electrophysiology}

A trained observer experienced in neurology and clinical neurophysiology (RP) performed the examinations. The observer was blinded to the results of clinical and ultrasound examinations. Electrical nerve stimulation and recording of compound action potentials were performed using an isolated stimulator controlled by an Epoch XP-system coupled to a PowerLab28T Signal Conditioner with integrated BioAmp (ADInstruments, Sydney, Australia). At the time of first and second assessments, the motor functions of the ulnar and the fibular nerves were tested by bilateral recording of compound muscle action potentials (CMAP) from the abductor digiti minimi and the extensor digitorum brevis muscles using surface electrodes. Amplitudes as well as proximal and distal latencies of CMAP were measured to calculate nerve conduction velocities. Sensory nerve function was tested by recording sensory nerve action potentials following antidromic stimulation of the superficial branches of the radial and the sural nerves.

\section{MUS Protocol}

A neurologic expert in MUS (UW) performed all ultrasound examinations and was blinded to the clinical, the electrophysiological, and the laboratory findings. A clinical ultrasound system (Aplio 300, Toshiba, Tokyo, Japan) equipped with a 4- to $14-\mathrm{MHz}$ linear array transducer was used for muscle ultrasound using the musculoskeletal ("MSK") preset. The ultrasound system settings including image contrast were kept constant during all examinations according to previous recommendations. ${ }^{19}$ Imaging depth and focus were adapted to optimally visualize region of interest. On days 3 and 10 after study inclusion, the following eight extremity muscles were assessed on MUS: bilateral biceps brachii, bilateral brachioradialis, bilateral quadriceps femoris, and bilateral tibialis anterior muscle. For each muscle, the echogenicity was rated on a four-grade scale according to the following criteria: grade 1 = normal echo-intensity and normal structure of the muscle and normal echosignal of adjacent bone with complete visualization of the highly echogenic corticalis; grade $2=$ increased echointensity and slightly altered structure of the muscle and normal echosignal of adjacent bone with complete visualization of the highly echogenic corticalis; grade 3 = increased echointensity and moderately altered structure of the muscle and altered echosignal of adjacent bone with incomplete visualization of the highly echogenic corticalis; and grade $4=$ increased echointensity and severely altered structure of the muscle and lost bone echo without visualization of the corticalis. ${ }^{19}$ The mean global muscle echogenicity score (GME) was calculated for each subject by summarizing the echogenicity grades of all eight muscles_—with a minimum score of 1 if all muscles were normal and increasing score (maximum 4) indicating more severe alteration of the muscles.

\section{Serum Markers of Inflammation and Endothelial Damage}

Serum concentrations of procalcitonin (PCT) were measured at the time of first and second clinical assessments using the Kryptor Compact Plus (Thermo Fisher Scientific, MA, USA). The serum concentrations of endothelial surface proteins e-selectin (CD62e) and syndecan-1 (CD138) and of interleukin-6 (IL-6) were measured according to the manufacturers' recommendations at both times of assessment using commercially available ELISA-kits that were purchased from Abcam, Cambridge, United Kingdom (e-selectin: ab11512; syndecan-1: ab46506; IL-6: ab46027). The GloMax platereader (Promega/Fitchburg, USA) was used for all fluorescence measurements.

\section{Healthy Control Group}

Healthy, age-matched volunteers recruited from personnel of the Institute of Physiology, University of Rostock, underwent the same assessments as the patients. The procedures, which were all performed in one session, comprised a neurological investigation including MRC-SS, BI and mRs, electrophysiology, MUS and measurement of IL-6, PCT, syndecan-1, and e-selectin serum concentration. Electrophysiology, MUS, and laboratory studies were performed with the same devices and settings that were used for patient examinations.

\section{Statistical Methods}

Comparison of normally distributed data was performed with the two-sided $t$-test and of non-normally distributed data with the Mann-Whitney $U$ test. All correlation analyses were performed using Pearson's $R$ for normally distributed and Spearman's $R$ for non-normally distributed data. Statistical significance was indicated by $p<0.05$. A Bonferroni correction was applied for multiple correlations involving the four laboratory parameters (IL-6, PCT, e-selectin, and syndecan-1), with $p<0.013$ indicating statistical significance. All statistical analyses were performed with IBM SPSS Statistics (Version 22, Chicago, IL, USA). 


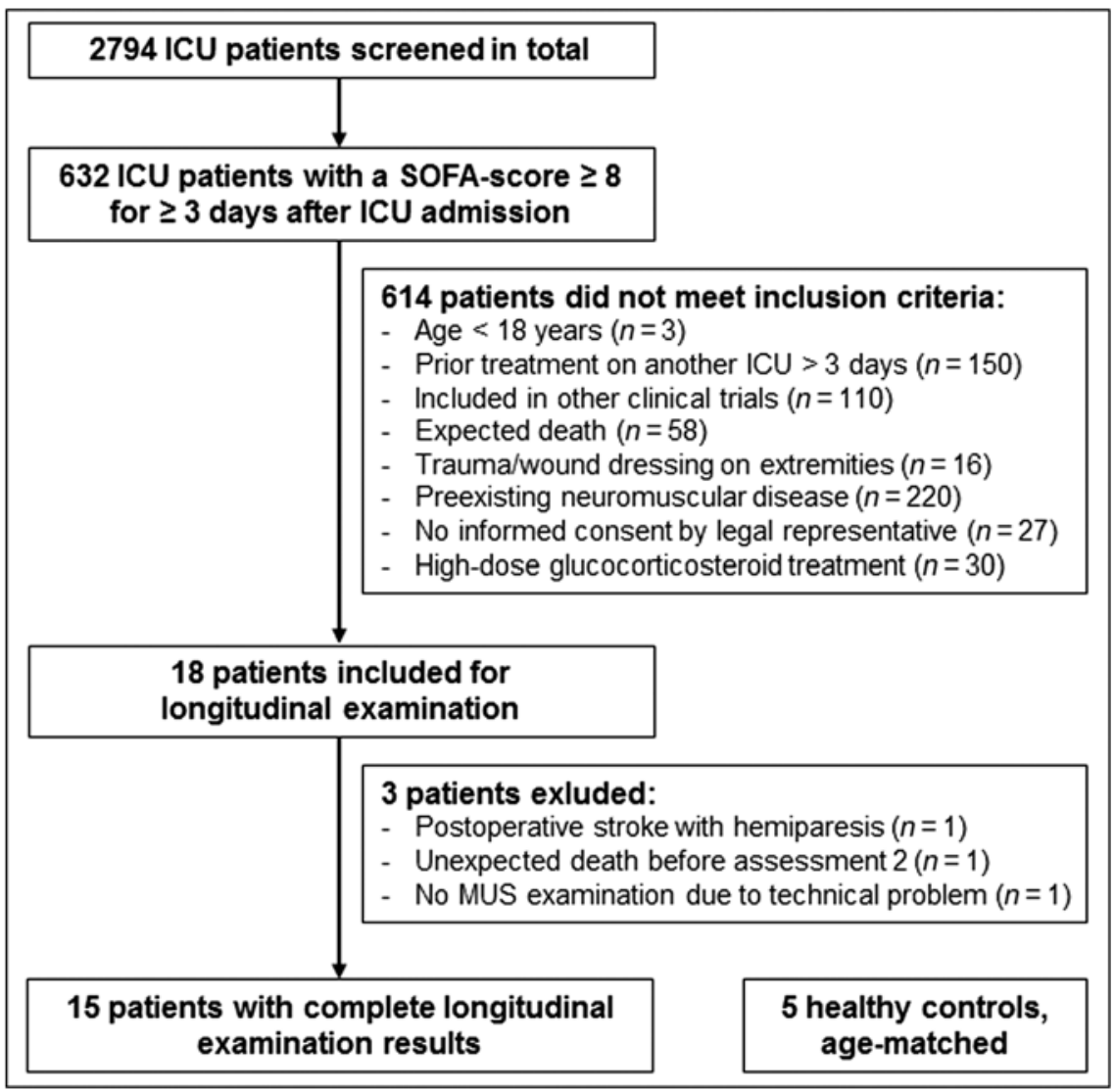

Figure 2: Patient enrollment. ICU, intensive care unit; SOFA, sequential organ failure assessment; MUS, muscular ultrasound.

\section{Results}

\section{Patient Characteristics and Long-Term Outcome for 100 Days}

Between March 2016 and March 2017, a total of 15 critically ill ICU patients and five healthy controls were studied (Figure. 2). The patient group comprised 11 men and 4 women. The mean age of patients was $58.9( \pm 15.1)$ years. The control group comprised three men and two women with a mean age of $49.8( \pm 10.3)$ years.

All control subjects survived until day 100 after study inclusion (mRS, 0). Two out of 15 patients died within 100 days (Table 1). The mean value on the mRS of the 13 survivors was $2.71( \pm 2.4)$ and was significantly higher in comparison to controls $(p=0.023)$.

\section{Clinical Assessment of ICU-AW}

All 15 patients were longitudinally assessed at two time points of assessment. At the time of assessment 1, four patients were assessable by MRC-SS (mean MRC-SS $51.5 \pm 6.0$ ) which resulted in the diagnosis of ICU-AW in one patient (MRC-SS 34). The MRC-SS was "unable-to-assess" in 11 participants. Eleven patients were mechanically ventilated.

At the time of assessment 2, the MRC-SS was assessable in 11 patients (mean MRC-SS of $44.7 \pm 5.5$ ). In four patients with unable-to-assess MRC-SS documentation, the neurologic examination gave strong evidence for ICU-AW as these patients showed symmetric and flaccid paresis according to the ICU-AW definition [2]. In total, 8 out of 15 patients were diagnosed with ICU-AW. Nine patients were mechanically ventilated (Table 2).
In the five control subjects, no signs of ICU-AW were observed by clinical assessment (Table 2).

\section{Electrophysiological Assessment in Patients and Controls}

The neurophysiological findings were within the physiological range for all control subjects (Table 3). All patients showed significantly reduced CMAP amplitudes indicating axonal neuropathy and/or myopathy. Conduction velocities were also reduced in patients but to a lesser extent than CMAP amplitudes. Alterations of motor nerve responses were consistently more pronounced in lower than in upper extremities. None of the patients had normal motor or sensory CAP (Supplementary Figure. S1).

\section{MUS to Assess ICU-AW}

Results from MUS were available from all 20 participants. The mean GME of the patient cohort was $2.31( \pm 0.41)$ at assessment 1 and did not decrease until assessment $2(2.21 \pm 0.41)$. The mean GME of $1.06( \pm 0.06)$ of the control subjects was lower in comparison to patients' GME at assessments $1(p=0.001)$ and $2(p=0.001)$. Typical examples of muscular echogenicity in a patient and a control subject are shown in Figure. 3.

Within the patients' cohort, the eight participants with ICUAW tended to larger GME at assessment $2(2.34 \pm 0.35)$ compared to the seven participants without ICU-AW (2.06 \pm 0.45 ; $p=0.089)$. Such a trend was not observed at the time of assessment 1. In comparison with the control subjects, the patients without ICU-AW ( $p=0.04)$ as well as the patients with ICU-AW $(p=0.03)$ showed higher GME (Figure. 4A). 
Table 1: Clinical characteristics and long-term outcome for 100 days

\begin{tabular}{|c|c|c|c|c|c|}
\hline Patient/study group & Age/gender & $\begin{array}{c}\text { Indication for ICU } \\
\text { admission }\end{array}$ & $\mathrm{BI} / \mathrm{mRs}$ prior to $\mathrm{ICU}$ & APACHE-II/Worst SOFA & Survival for 100 days \\
\hline $1 / \mathrm{p}$ & $34 / \mathrm{m}$ & Surgical emergency/trauma & $100 / 0$ & $17 / 11$ & Yes \\
\hline $2 / \mathrm{p}$ & $44 / \mathrm{m}$ & Surgical emergency/trauma & $100 / 0$ & $25 / 16$ & Yes \\
\hline $3 / \mathrm{p}$ & $64 / \mathrm{m}$ & Elective surgery & $100 / 0$ & $8 / 10$ & Yes \\
\hline $4 / \mathrm{p}$ & $59 / \mathrm{f}$ & Surgical emergency/trauma & $100 / 0$ & $26 / 13$ & Yes \\
\hline $5 / \mathrm{p}$ & $58 / \mathrm{f}$ & Surgical emergency/trauma & $100 / 0$ & $9 / 21$ & No \\
\hline $6 / \mathrm{p}$ & $68 / \mathrm{f}$ & Surgical emergency/trauma & $100 / 0$ & $24 / 21$ & Yes \\
\hline $7 / \mathrm{p}$ & $65 / \mathrm{m}$ & Elective surgery & $100 / 0$ & $27 / 10$ & Yes \\
\hline $8 / \mathrm{p}$ & $57 / \mathrm{m}$ & Elective surgery & $100 / 0$ & $21 / 11$ & Yes \\
\hline $9 / \mathrm{p}$ & $76 / \mathrm{m}$ & Surgical emergency/trauma & $100 / 0$ & $28 / 9$ & Yes \\
\hline $10 / \mathrm{p}$ & $69 / \mathrm{m}$ & Elective surgery & $100 / 0$ & $28 / 14$ & Yes \\
\hline $11 / \mathrm{p}$ & $38 / \mathrm{m}$ & Surgical emergency/trauma & $100 / 0$ & $22 / 10$ & Yes \\
\hline $12 / \mathrm{p}$ & $81 / \mathrm{m}$ & Elective surgery & $100 / 0$ & $30 / 13$ & Yes \\
\hline $13 / \mathrm{p}$ & $81 / \mathrm{f}$ & Surgical emergency/trauma & $95 / 1$ & $29 / 13$ & No \\
\hline $14 / \mathrm{p}$ & $42 / \mathrm{m}$ & Elective surgery & $100 / 0$ & $27 / 19$ & Yes \\
\hline $15 / \mathrm{p}$ & $48 / \mathrm{m}$ & Surgical emergency/trauma & $100 / 0$ & $28 / 13$ & Yes \\
\hline $16 / \mathrm{c}$ & $54 / \mathrm{f}$ & n.a. & $100 / 0$ & n.a. & Yes \\
\hline $17 / \mathrm{c}$ & $33 / \mathrm{m}$ & n.a. & $100 / 0$ & n.a. & Yes \\
\hline $18 / \mathrm{c}$ & $50 / \mathrm{m}$ & n.a. & $100 / 0$ & n.a. & Yes \\
\hline $19 / \mathrm{c}$ & $51 / \mathrm{f}$ & n.a. & $100 / 0$ & n.a. & Yes \\
\hline $20 / \mathrm{c}$ & $61 / \mathrm{m}$ & n.a. & $100 / 0$ & n.a. & Yes \\
\hline
\end{tabular}

APACHE-II, acute physiology and chronic health evaluation; BI, Barthel index; c, control; f, female; ICU, intensive care unit; m, male; mRs, modified Rankin scale; p, patient; SOFA, sequential organ failure assessment.

Table 2: Clinical assessment of patients and controls

\begin{tabular}{|c|c|c|c|c|}
\hline & $\begin{array}{l}\text { Assessment } 1 \text { patient group } \\
(n=15)\end{array}$ & $\begin{array}{c}\text { Assessment } 1 \text { Control group } \\
(n=5)\end{array}$ & $\begin{array}{c}\text { Assessment } 2 \text { Patient group } \\
(n=15)\end{array}$ & $\begin{array}{l}\text { Assessment } 2 \text { control group } \\
(n=5)\end{array}$ \\
\hline Mechanical ventilation $(n)$ & 11 & 0 & 9 & $\begin{array}{l}\text { n.a. } \\
\end{array}$ \\
\hline MRC-SS assessable $(n)$ & 4 & 5 & 11 & n.a. \\
\hline Mean $( \pm$ SD) MRC-SS $(n)$ & $51.5( \pm 6.0)$ & $60( \pm 0)$ & $44.7( \pm 5.5)$ & n.a. \\
\hline $\begin{array}{l}\text { ICU-AW diagnosed according to } \\
\text { criteria }(n)[2]\end{array}$ & 1 & 0 & 8 & n.a. \\
\hline
\end{tabular}

ICU-AW, intensive care unit-acquired weakness; MRC-SS, muscular research council sum score; n.a., not applicable; SD, standard deviation.

We observed a different longitudinal development of the GME values between patients with and without ICU-AW over time (Figure. 4B). The GME values of patients with ICU-AW did not decrease from assessment 1 (mean GME, $2.33 \pm 0.37$ ) to assessment 2 (mean GME, $2.33 \pm 0.36$ ). In contrast, mean GME values significantly decreased in patients without ICU-AW from assessment $1(2.30 \pm 0.48)$ to assessment $2(2.06 \pm 0.45 ; p=0.027)$.

\section{Serum Markers of Inflammation and Endothelial Damage}

The serum levels of PCT, IL-6, e-selectin, and syndecan-1 at assessment 1 were significantly higher in patients than in controls (Table 4). Biomarker measurements at assessments 1 and 2 revealed a decrease over time of IL-6 $(p=0.003)$ and PCT $(p=0.001)$ but not of e-selectin and syndecan-1 levels (Figure. 5).

\section{Relationship Between Serum Markers and Muscle Echogenicity}

At the time of patients' assessments 1 and 2, no correlation was found between GME and any of the serum biomarkers measured at the same time of assessment. By contrast, syndecan-1 measured at assessment 1 correlated with higher GME detected at assessment $2(r=0.63, p=0.012)$.

\section{Prognostic Value of Ultrasound and Serum Biomarker Findings}

In the patient group, higher GME observed at assessment 2 was related to poorer clinical long-term outcome represented by higher mRS values at day $100(r=0.58, p=0.04)$. The sum of patients' GME at assessments 1 and 2 correlated even stronger 
Table 3: Parameters of motor and sensory nerve functions in patients and controls

\begin{tabular}{|c|c|c|c|c|c|c|c|c|}
\hline & & Controls & SD & Obtainable & Patients & SD & Obtainable & $p$-value \\
\hline \multirow[t]{4}{*}{ Motor } & CMAP UE (mV) & 13.365 & 2.4 & $10 / 10$ & 4.6 & 2.8 & $59 / 60$ & $<0.0001$ \\
\hline & CV UE (m/s) & 60 & 5.5 & $10 / 10$ & 54 & 16 & $56 / 60$ & 0.003 \\
\hline & CMAP LE (mV) & 7.3 & 2.2 & $10 / 10$ & 1.7 & 2 & $50 / 60$ & $<0.0001$ \\
\hline & CV LE $(\mathrm{m} / \mathrm{s})$ & 55 & 4.6 & $10 / 10$ & 44 & 13.5 & $45 / 60$ & 0.018 \\
\hline \multirow[t]{4}{*}{ Sensory } & SNAP UE (mV) & 48.7 & 15.5 & $10 / 10$ & 13.8 & 5.6 & $41 / 60$ & $<0.0001$ \\
\hline & CV UE (m/s) & 54 & 10.5 & $10 / 10$ & 53 & 11.5 & $41 / 60$ & 0.6 \\
\hline & SNAP LE $(\mathrm{mV})$ & 19.6 & 3.6 & $10 / 10$ & 9.9 & 6.1 & $20 / 60$ & 0.0004 \\
\hline & CV LE $(\mathrm{m} / \mathrm{s})$ & 55 & 8.8 & $10 / 10$ & 47 & 10 & $20 / 60$ & 0.04 \\
\hline
\end{tabular}

Mean values of both assessments are given for the patient group. Arithmetic means from right and left side measurements are given in all cases. Whereas all patients but one had MSAP reductions at extensor digitorum brevis at first assessment, MSAP from abductor digiti minimi were still within the physiologic range in four patients. Furthermore, extensor digitorum brevis responses in all patients were further reduced at the time of second assessment, MSAP recorded from abductor digiti minimi showed at least partial recovery in five patients. The amplitudes of compound action potentials from sensory nerves were also significantly reduced when compared to values obtained in control subjects. Especially SNAP from sural nerves were reduced and not detectable at all in 20 from 60 measurements. CMAP, compound muscle action potential; CV, conduction velocity; UE, upper extremity; LE, lower extremity; SD, Standard deviation; SNAP, sensory nerve action potential.

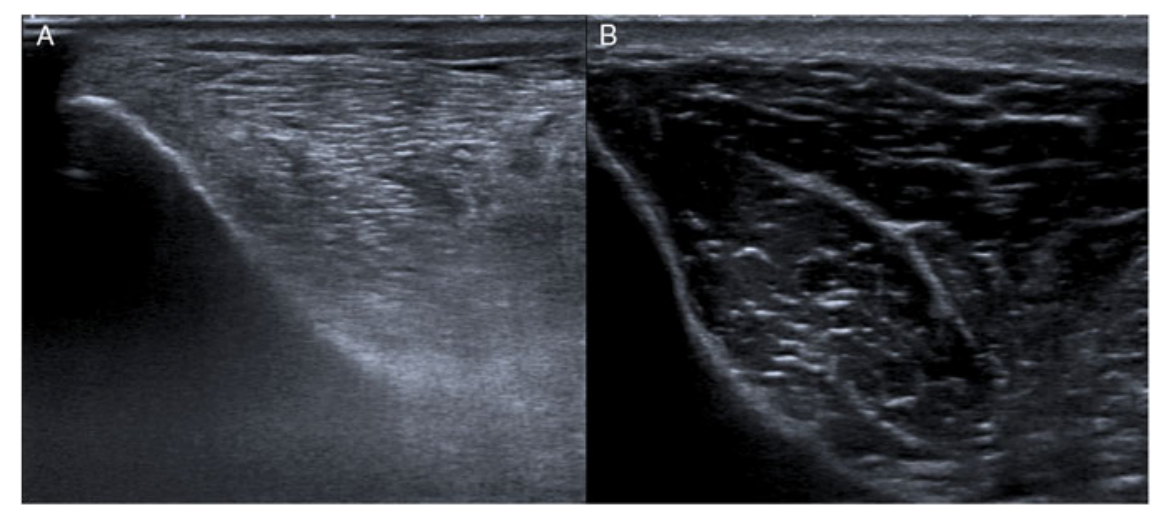

Figure 3: Muscular ultrasound images of increased and normal echogenicity. A, Right tibialis anterior muscle of a patient (case \#5) showing increased echogenicity (grade 3) at the time of assessment 1. B, Right tibialis anterior muscle of a control subject (case \#16) showing normal muscle echogenicity (grade 1).
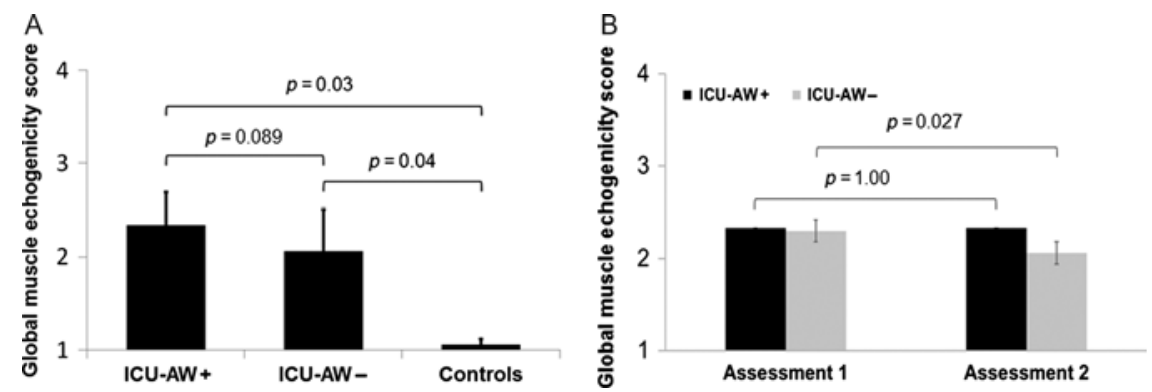

Figure 4: GME scores in patients and controls. A, Different GME scores between ICU-AW+ and ICU-AW- patients (as classified at assessment 2) compared to controls. B, Different longitudinal development of GME scores between ICU-AW+ and ICU-AW-patients (as classified at assessment 2) over time. ICU-AW+, intensive care unit-acquired weakness present; $I C U-A W$-, intensive care unit-acquired weakness not present; n.s., non-significant.

with the 100-day mRs $(r=0.67 ; p=0.013$, Figure. 6). Patients with a summed GME (assessments 1 and 2 ) of $>4.5$ were more likely to have an unfavorable outcome ( $\mathrm{mRs}>2)$ compared to those with a summed GME of $<4.5$ (OR, 12.5; 95\% CI, $0.84-186 ; p=0.048)$.
Among the investigated biomarkers, only serum PCT levels were related to 100-day outcome. PCT levels measured at assessment 1 were lower in patients with an $\mathrm{mRs}$ of $\leq 2$ compared to patients with an $\mathrm{mRS}$ of $>2$ at day 100 (mean PCT $3.4 \pm 6.1 \mathrm{ng} / \mathrm{ml} \mathrm{vs}$. $26.2 \pm 32.2 \mathrm{ng} / \mathrm{ml}, p=0.014)$. PCT levels from both time 
Table 4: Values of biomarkers of inflammation and endothelial damage in patients and controls

\begin{tabular}{l|c|c|c|c|c}
\hline Biomarker & $\begin{array}{c}\text { Assessment 1 patients, } \\
\text { mean } \pm \text { SD }\end{array}$ & $\begin{array}{c}\text { Assessment 2 patients, } \\
\text { mean } \pm \text { SD }\end{array}$ & Assessment 1 vs. 2, $\boldsymbol{p}$ value & $\begin{array}{c}\text { Assessment 1 controls, } \\
\text { mean } \pm \text { SD }\end{array}$ & $\begin{array}{c}\text { Patients vs. controls, } \\
\boldsymbol{p} \text { value }\end{array}$ \\
\hline PCT $(\mathrm{ng} / \mathrm{ml})$ & $15.4 \pm 26.0$ & $2.1 \pm 3.3$ & 0.001 & $<0.1 \pm 0$ & 0.001 \\
\hline IL-6 $(\mathrm{pg} / \mathrm{ml})$ & $93.2 \pm 71.1$ & $28.9 \pm 40.5$ & 0.003 & $9.6 \pm 0.9$ & 0.013 \\
\hline e-selectin $(\mathrm{ng} / \mathrm{ml})$ & $364.7 \pm 360.7$ & $232.0 \pm 136.5$ & 0.173 & $66.5 \pm 87.0$ & 0.006 \\
\hline syndecan-1 $(\mathrm{ng} / \mathrm{ml})$ & $263.8 \pm 108.0$ & $264.3 \pm 101.6$ & 0.427 & $6.0 \pm 2.6$ & 0.001 \\
\hline
\end{tabular}

IL-6, interleukin-6; PCT, procalcitonin; SD, standard deviation.
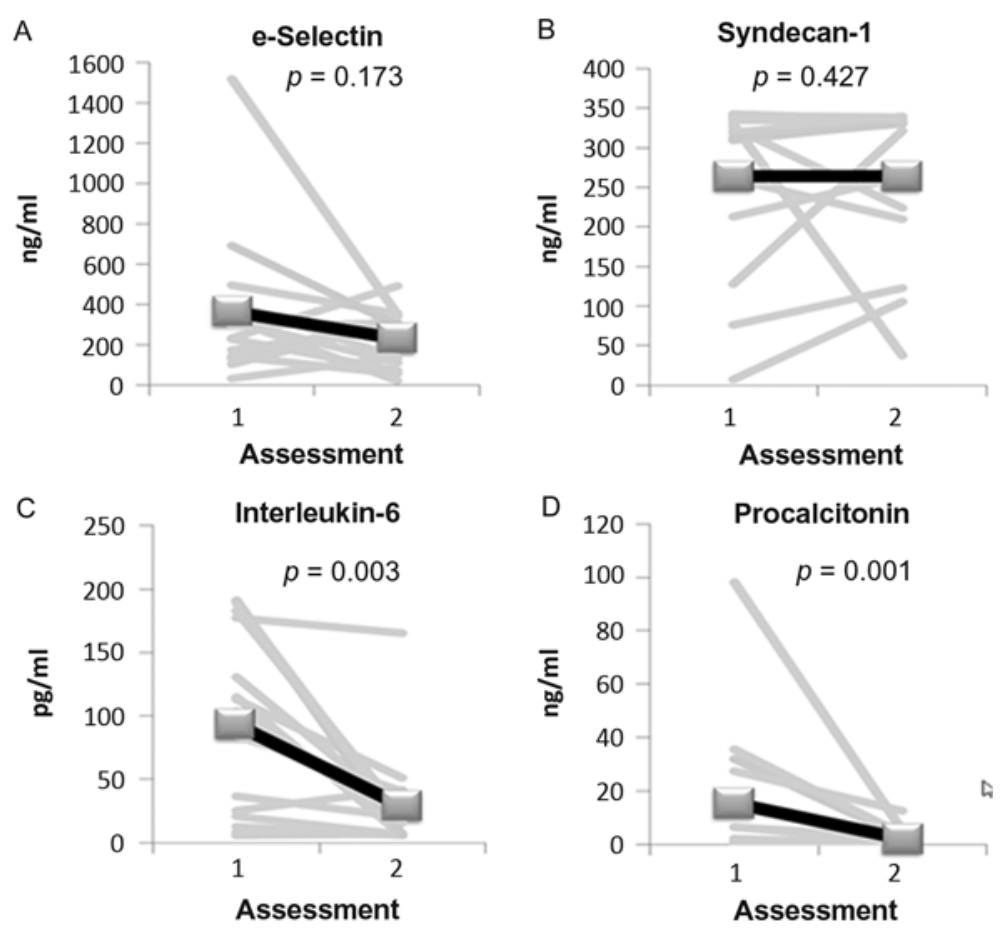

Figure 5: Longitudinal development of serum biomarkers in the patient group. $I L-6$, interleukin-6; PCT, procalcitonin.

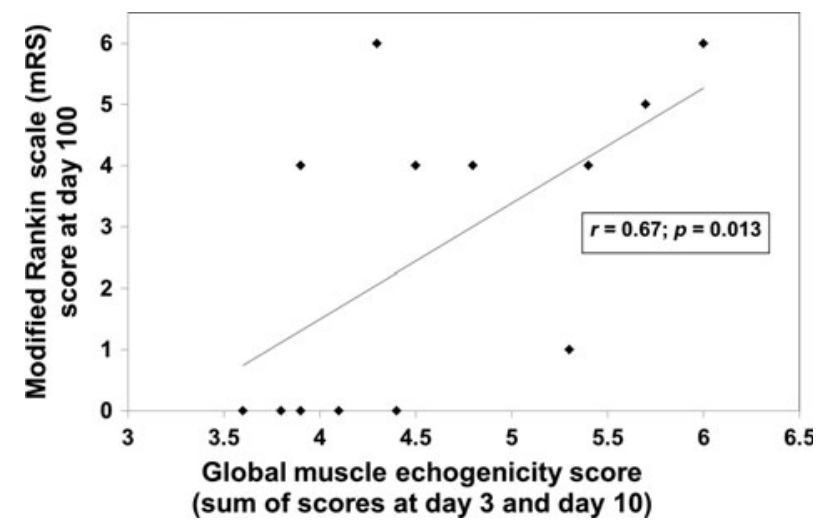

Figure 6: Correlation of GME scores with the 3-month patient outcome.

points of assessment correlated with $\mathrm{mRS}$ values at day 100 (PCT assessment 1, $r=0.64, p=0.013$; PCT at assessment 2, $r=0.65, p=0.012$ ). However, PCT levels did not improve the discrimination of patients with favorable versus unfavorable outcome. If the GME value at assessment 2 was multiplied by the PCT serum level $[\mathrm{ng} / \mathrm{ml}]$ at assessment 1 , a resulting value of $>3$ discriminated patients with unfavorable outcome (mRs $>2$ at day 100) from those with favorable outcome with a sensitivity of $100 \%$ and a specificity of $83 \%$ (Fisher's exact test, $p=0.005)$.

\section{Discussion}

This prospective clinical study was performed to evaluate the relevance of increased muscle echogenicity detected by MUS and elevated markers of inflammation and endothelial damage in critically ill patients for diagnosing ICU-AW and for predicting the 3-month outcome. The present data suggest that abnormal MUS findings may support the diagnosis of ICU-AW. Furthermore, abnormal MUS findings are clearly predictive of the 3-month outcome, especially if MUS findings are combined with PCT serum levels. Increased muscle echogenicity was related to elevated syndecan-1 serum levels, which underlines the idea of inflammatory processes leading to abnormal muscle echogenicity. 
The incidence rate of 53\% of patients diagnosed with ICU-AW in our study was comparable to previous reports. ${ }^{1,3,17,29}$ Difficulties to rapidly diagnose ICU-AW in a majority of sedated and mechanically ventilated critically ill patients, as confirmed in the present study, are of importance for ICU physicians. ${ }^{2,17,30}$ Clinical evaluation of the critically ill by using the MRC-SS alone could not detect ICU-AW, particularly at the time of assessment 1 . This confirms the necessity for further diagnostic tools. In order to detect even subclinical disturbances in the neuromuscular function and to rule out other neuromuscular diseases like acute inflammatory demyelinating polyneuropathy, time consuming nerve conduction studies were performed. Our electrophysiological results gave some evidence for neuromuscular disturbances in our patients, but we were not able to identify patients at risk for ICU-AW early during the phase of critical illness. More invasive techniques like needle myography would have been necessary to overcome the shortcomings of surface neuromyography. ${ }^{31}$

Therefore, MUS as a non-invasive and widely available technique and body fluid biomarkers are of high clinical interest. Several studies reported on the diagnostic relevance of MUS for the diagnosis of muscular dysfunction. ${ }^{17,19,20,22,32-34}$ Heckmatt et al. reported increased muscle echogenicity in muscle disease and graded the extent of echogenicity on a four-point scale. ${ }^{22}$ More recent studies focused on muscle echogenicity in critically ill patients. ${ }^{15,19}$ The etiology of increased echogenicity is not fully understood. ${ }^{16}$ Muscle edema due to capillary leakage in the acute stage of sepsis and excessive fluid resuscitation is discussed. ${ }^{19}$ A recent comparison of histological specimens of the vastus lateralis muscle and MUS demonstrated a correlation of increased muscle echogenicity with myofascial inflammation characterized by edema, neutrophil infiltration, fibrin deposition, and accompanying myofiber necrosis. ${ }^{23}$ Our results lend further support to an inflammatory, possibly immune-mediated pathogenesis of ICU-AW in critically ill patients. ${ }^{3,10,12,13}$ Syndecan-1 as a marker of endothelial damage significantly correlated with increased GME values in our study. This is highly interesting from a pathophysiological point of view as it might direct towards a systemic inflammatory stimulus with a local endothelial response at the muscular membranes of skeletal muscles in ICU-AW. The pure clinical design of the present study is insufficient to give proof for this hypothesis. Further investigations employing sequential ultrasound measurements in combination with muscle biopsies and immunohistochemistry could elucidate these suspected immune-mediated pathways. ${ }^{23}$

Our data show for the first time that the combination of two easily assessable diagnostic features, i.e. MUS and serum analyte measures (here, of early PCT levels), may improve the identification of patients at risk for unfavorable outcome. Provided the confirmation of our findings in large scale studies, this approach might enable in near future the selection of such patients at risk for more specific, early anti-inflammatory treatment strategies.

Although we performed a longitudinal study with multimodal diagnostics, several limitations have to be mentioned. The low number of patients in this pilot observation represents a shortcoming, although these patients were intensively assessed by multimodal diagnostics. Our study especially reflects the problem of limited feasibility of a reliable clinical examination due to sedation and mechanical ventilation. We had a rather high number of patients being unable to be assessed on the MRC-SS, which was otherwise a reason to perform the present study. Nevertheless, our findings of increased muscle echogenicity, PCT, and syndecan-1 levels measured during the early phase of critical illness may promote its broader use by intensivists as our data indicate longterm prognostic relevance. Biomarker measurements and MUS are widely available tools. They might be helpful to close the gap between the insufficient clinical examination of patients on risk for ICU-AW at early stages of critical illness and the high incidence of ICU-AW during the further course of disease.

\section{Conclusion}

Pure clinical and electrophysiological evaluations of ICU patients are insufficient to diagnose ICU-AW in the early courses of critical illness. Increased echogenicity detected by MUS is associated with critical illness, long-term outcome of survivors, and by trend with ICU-AW. The longitudinal assessment of echogenicity over time might help to discriminate between patients with and without ICU-AW during the course of critical illness. Syndecan-1 as a marker of endothelial damage is related to increased muscle echogenicity and might play an important role in the pathophysiology of ICU-AW. The combination of two easily assessable diagnostic features, i.e. MUS and serum analyte measures, may improve the identification of patients at risk for unfavorable long-term outcomes. Further prospective clinical studies and experimental data are warranted to analyze the link between inflammation, endothelial damage and increased echogenicity detected by MUS in patients with ICU-AW.

\section{SUPPLEMENTARY Material}

To view supplementary material for this article, please visit https://doi.org/10.1017/cjn.2018.390.

\section{ACKNOWLEDGements}

The authors thank Georg Richter (Department of Anesthesiology and Intensive Care Medicine) and Karim Zanaty (Institute of Physiology) for organizational study assistance.

The medical faculty of the University of Rostock supported the study with a grant from the "FORUN" research program (grant number 889006).

\section{STATEMENT OF Authorship}

JE and RP conducted the study. JE, RP, and UW wrote the manuscript. MS and DAR edited the manuscript and provided conceptual advice. UW and SR performed all ultrasound examinations. RP performed all electrophysiological examinations. JE screened all ICU patients and performed all clinical examinations in the study participants. All authors contributed significantly to the scientific content of the manuscript and approved its final version.

\section{Disclosures}

Dr. Walter reports grants and personal fees from Merz Pharma, personal fees from Bristol-Myers Squibb, personal fees from Daiichi Sankyo, personal fees from Bayer Vital, personal fees from Pfizer, other from Elsevier Press, other from Georg Thieme Verlag, and other from European Journal of Ultrasound, outside the submitted work. The remaining authors have no conflicts of interest to declare. 


\section{REFERENCES}

1. Stevens RD, Dowdy DW, Michaels RK, Mendez-Tellez PA, Pronovost PJ, Needham DM. Neuromuscular dysfunction acquired in critical illness: a systematic review. Intensive Care Med. 2007;33:1876-91.

2. Stevens RD, Marshall SA, Cornblath DR, et al. A framework for diagnosing and classifying intensive care unit-acquired weakness. Crit Care Med. 2009;37:299-308.

3. Witteveen E, Wieske L, van der Poll T, et al. Increased early systemic inflammation in ICU-acquired weakness; A prospective observational cohort study. Crit Care Med. 2017;45:972-9.

4. Latronico N, Bolton CF. Critical illness polyneuropathy and myopathy: a major cause of muscle weakness and paralysis. Lancet Neurol. 2011;10:931-41.

5. Weber-Carstens S, Deja M, Koch S, et al. Risk factors in critical illness myopathy during the early course of critical illness: a prospective observational study. Crit Care. 2010;14:R119.

6. Langhans C, Weber-Carstens S, Schmidt F, et al. Inflammationinduced acute phase response in skeletal muscle and critical illness myopathy. PLoS One. 2014;9:e92048.

7. Farhan H, Moreno-Duarte I, Latronico N, Zafonte R, Eikermann M. Acquired muscle weakness in the surgical intensive care unit: nosology, epidemiology, diagnosis, and prevention. Anesthesiology. 2016;124:207-34.

8. Kress JP, Hall JB. ICU-acquired weakness and recovery from critical illness. N Engl J Med. 2014;370:1626-35.

9. Marshall JC. Inflammation, coagulopathy, and the pathogenesis of multiple organ dysfunction syndrome. Crit Care Med. 2001;29(Suppl 7):99-106.

10. Chelazzi C, Villa G, Mancinelli P, De Gaudio AR, Adembri C. Glycocalyx and sepsis-induced alterations in vascular permeability. Crit Care. 2015;19:26.

11. Xing K, Murthy S, Liles WC, Singh JM. Clinical utility of biomarkers of endothelial activation in sepsis-a systematic review. Crit Care. 2012;16:R7.

12. Fenzi F, Latronico N, Refatti N, Rizzuto N. Enhanced expression of E-selectin on the vascular endothelium of peripheral nerve in critically ill patients with neuromuscular disorders. Acta Neuropathol. 2003;106:75-82.

13. Latronico N, Peli E, Botteri M. Critical illness myopathy and neuropathy. Curr Opin Crit Care. 2005;11:126-32.

14. Shepherd S, Batra A, Lerner DP. Review of critical illness myopathy and neuropathy. Neurohospitalist. 2017;7:41-8.

15. Cartwright MS, Kwayisi G, Griffin LP, et al. Quantitative neuromuscular ultrasound in the intensive care unit. Muscle Nerve. 2013;47:255-9.

16. Connolly B, MacBean V, Crowley C, et al. Ultrasound for the assessment of peripheral skeletal muscle architecture in critical illness: a systematic review. Crit Care Med. 2015;43: 897-905.

17. Witteveen E, Sommers J, Wieske L, et al. Diagnostic accuracy of quantitative neuromuscular ultrasound for the diagnosis of intensive care unit-acquired weakness: a cross-sectional observational study. Ann Intensive Care. 2017;7:40.
18. Bunnell A, Ney J, Gellhorn A, Hough CL. Quantitative neuromuscular ultrasound in intensive care unit-acquired weakness: a systematic review. Muscle Nerve. 2015;52:701-8.

19. Grimm A, Teschner U, Porzelius C, et al. Muscle ultrasound for early assessment of critical illness neuromyopathy in severe sepsis. Crit Care. 2013;17:R227.

20. Grimm A, Heiling B, Schumacher U, Witte OW, Axer H Ultrasound differentiation of axonal and demyelinating neuropathies. Muscle Nerve. 2014;50:976-83.

21. Parry SM, El-Ansary D, Cartwright MS, et al. Ultrasonography in the intensive care setting can be used to detect changes in the quality and quantity of muscle and is related to muscle strength and function. J Crit Care. 2015;30:1151.e9-14.

22. Heckmatt JZ, Leeman S, Dubowitz V. Ultrasound imaging in the diagnosis of muscle disease. J Pediatr. 1982;101:656-60.

23. Puthucheary ZA, Phadke R, Rawal J, et al. Qualitative ultrasound in acute critical illness muscle wasting. Crit Care Med. 2015;43: 1603-11.

24. Vincent JL, Moreno R, Takala J, et al. The SOFA (Sepsis-related organ failure assessment) score to describe organ dysfunction/ failure. On behalf of the Working Group on Sepsis-related problems of the European Society of Intensive Care Medicine. Intensive Care Med. 1996;22:707-10.

25. Knaus WA, Draper EA, Wagner DP, Zimmerman JE. APACHE II: a severity of disease classification system. Crit Care Med. 1985; 13:818-29.

26. Khanal K, Bhandari SS, Shrestha N, Acharya SP, Marhatta MN. Comparison of outcome predictions by the Glasgow coma scale and the full outline of unresponsiveness score in the neurological and neurosurgical patients in the intensive care unit. Indian J Crit Care Med. 2016;20:473-6.

27. Sessler CN, Gosnell MS, Grap MJ, et al. The Richmond agitationsedation scale: validity and reliability in adult intensive care unit patients. Am J Respir Crit Care Med. 2002;166:1338-44.

28. Villa P, Pintado MC, Luján J, et al. Functional status and quality of life in elderly intensive care unit survivors. J Am Geriatr Soc. 2016;64:536-42.

29. De Jonghe B, Sharshar T, Lefaucheur JP, et al. Paresis acquired in the intensive care unit: a prospective multicenter study. JAMA 2002;288:2859-67.

30. Vanpee G, Hermans G, Segers J, Gosselink R. Assessment of limb muscle strength in critically ill patients: a systematic review. Crit Care Med. 2014;42:701-11.

31. Weber-Carstens S, Koch S, Spuler S, et al. Nonexcitable muscle membrane predicts intensive care unit-acquired paresis in mechanically ventilated, sedated patients. Crit Care Med. 2009;37: 2632-7.

32. Grimm A, Décard BF, Axer H, Fuhr P. The ultrasound pattern sum score - UPSS. A new method to differentiate acute and subacute neuropathies using ultrasound of the peripheral nerves. Clin Neurophysiol. 2015;126:2216-25.

33. Walker FO. Neuromuscular ultrasound. Neurol Clin. 2004;22: 563-90.

34. Walker FO, Cartwright MS, Wiesler ER, Caress J. Ultrasound of nerve and muscle. Clin Neurophysiol. 2004;115:495-507. 